\title{
Influence of numerical schemes on statistical properties of computed charged particle trajectories in turbulent electromagnetic fields
}

\author{
C.C. Lalescu*,a,b,1, B. Teaca ${ }^{\mathrm{c}}$, D. Carati $^{\mathrm{a}}$ \\ ${ }^{a}$ Statistical and Plasma Physics, Université Libre de Bruxelles, Campus Plaine, CP 231, B-1050 \\ Brussels, Belgium \\ ${ }^{b}$ Faculty of Physics, University of Craiova, A.I. Cuza 13, 200585 Craiova, Romania \\ ${ }^{c}$ Centre de Recherches en Physique des Plasmas, Science de Base, Ecole Polytechnique Federale de \\ Lausanne, Station 13, Building PPB, CH-1015 Lausanne, Switzerland
}

\begin{abstract}
A class of numerical schemes is developed for the study of charged particle transport in complex stationary electromagnetic fields and tested for fields obtained from a numerical solution of the magneto-hydrodynamic equation. The performances of these schemes are evaluated by analyzing the conservation of energy and the statistical properties of the trajectories. Energy conservation is affected by the interpolation technique used to estimate the field value at the particle position. However, the particle transport properties are more robust, except in the limit of low energy when a significant fraction of the particles are trapped.
\end{abstract}

Key words: Spline interpolation, Charged particle transport, Turbulent electromagnetic fields

PACS: 47.27.T-, 02.60.Ed, 02.60.Pn, 45.50.-j

\section{Introduction}

The integration of individual trajectories of charged particles in turbulent electromagnetic fields is required in a number of different contexts. Some well known examples are those of impurities in fusion plasma devices [1], pseudo-particles in particle in cell (PIC) codes [2,3] and particles in astrophysical plasmas [4, 5]. A charged particle in an electromagnetic field with a broad spectrum is expected to experience a very complex motion and the numerical integration of its trajectory is definitely not straightforward [6]. These trajectories have a number of global properties that usually correspond to physical constraints and that must be reproduced by the solver as accurately as possible. For

\footnotetext{
${ }^{*}$ Corresponding author. Tel: +1.410.516.6538, Fax: +1.410.516.7459

Email addresses: clalescu@ulb.ac.be (C.C. Lalescu), bogdan.teaca@epfl.ch (B. Teaca), dcarati@ulb.ac.be (D. Carati)

${ }^{1}$ Present address: Department of Applied Mathematics \& Statistics, 3400 North Charles Street, The Johns Hopkins University, Baltimore, Maryland 21218-2682, USA 
instance, the position of trapped particles is confined to a given domain in real space. Similarly, the velocity of ballistic particles is confined to a given domain in velocity space. Generally, the particle phase space will be separated into invariant regions of different behaviour, and particles cannot pass the boundaries between these regions. In numerical approximations of the dynamical system that describes the particle dynamics, the errors range from deformations of these regions to their complete destruction.

The global numerical approaches used for tracking particles that interact with a complex discretized field can be separated into two pieces. First, a time integration scheme is used to advance the particle position and velocity. It is usually a generic solver for integrating equations of motion as described in $[7,8,9]$. Second, a spatial interpolation scheme is needed to get the field value at the particle position. Indeed, electric and magnetic fields are typically known on a discrete grid and their values at arbitrary positions must be interpolated $[10,11]$. The two schemes do interact: the properties of the interpolation scheme have a strong influence on the effective properties of the solver, [12]. A more rigurous view is that a given interpolation scheme outputs a particular set of electromagnetic fields and the solver gives different results for different fields. In [13] it is shown that the use of cubic instead of linear interpolations may lead to different overall statistical properties for the particle transport. This naturally raises the question of the accuracy of the interpolation scheme.

Another important aspect is the efficiency of the numerical approach. The complexity of charged particle trajectories in the presence of turbulent electromagnetic fields usually imposes the simulation of a large particle ensemble in order to obtain reasonably converged statistics. In this case, high order time integration schemes that allow for better error control appear to be globally more efficient despite their cost per time step being higher. However, the use of high order time integration schemes requires in turn the use of high order spline interpolations [12].

The present study is focused on the transport of charged particles by stationary turbulent electromagnetic fields derived from a nonlinear magnetohydrodynamic simulation. The details of the numerical experiments are discussed in Section 2. For the stationary fields considered here, the total, kinetic plus potential, energy of the particle is conserved which implies a division of the phase space into constant energy manifolds. The numerical schemes used to follow the particle trajectories are briefly reviewed in Section 3. Ideally, the numerical simulation should preserve the particle energy exactly. It is shown in Section 4 how the energy error is affected by the use of different interpolation techniques. Moreover, the influence of the numerical schemes on the displacement and velocity statistics is discussed in detail. Interestingly, although the error on the energy conservation is shown to depend significantly on the interpolation scheme, these schemes have almost no influence on the probability distribution functions of both the velocity and the displacement of the particles. There is however one notable exception. In the limit of low energy, a significant fraction of the particle are trapped and linear interpolation schemes appear to be unable to reproduce all the features of the trajectory statistics. In Section 5 a brief investigation of transport by evolving MHD turbulence is discussed. Transport in MHD turbulence is shown to be a very different problem than that of transport by complex electrostatic fields. 


\section{Theoretical framework}

The evolution equation for the trajectory of non-relativistic charged particles with a mass $m$ and a charge $q$ are given by:

$$
\begin{aligned}
\partial_{t} \mathbf{r} & =\mathbf{v} \\
\partial_{t} \mathbf{v} & =\frac{q}{m}(\mathbf{E}(\mathbf{r})+\mathbf{v} \times \mathbf{B}(\mathbf{r}))
\end{aligned}
$$

where $\mathbf{r}$ and $\mathbf{v}$ are the position and the velocity of the particle and $\mathbf{E}(\mathbf{r})$ and $\mathbf{B}(\mathbf{r})$ are the electric and magnetic fields observed at the particle position. In studies for which the present work is most relevant, these fields are obtained by solving the magnetohydrodynamic equation. It is then common practice to introduce the Alfvén units $\left(\mathbf{b}=\mathbf{B} / \sqrt{\rho \mu_{0}}\right.$ and $\left.\mathbf{e}=\mathbf{E} / \sqrt{\rho \mu_{0}}\right)$, so that the "magnetic field" $\mathbf{b}$ has the dimension of a velocity, and the "electric field" e the dimension of a velocity square. Here $\rho$ is the density of the conductive fluid (which will be assumed to be a constant in space and time) and $\mu_{0}$ is the magnetic permeability of free space. In that case, the particle velocity equation is expressed as follows:

$$
\partial_{t} \mathbf{v}=\frac{1}{\ell}(\mathbf{e}(\mathbf{r})+\mathbf{v} \times \mathbf{b}(\mathbf{r}))
$$

where $\ell$ is a length scale that characterises the particle trajectory:

$$
\ell=\frac{m}{q \sqrt{\rho \mu_{0}}}
$$

This length scale is actually the Larmor radius of a particle that would move in a constant magnetic field $\mathbf{b}$ with $v_{\perp}=|\mathbf{b}|$ (here $v_{\perp}$ is the component of the velocity perpendicular to $\mathbf{b})$.

The electric and magnetic fields are obtained by solving the incompressible magnetohydrodynamic (MHD) equations:

$$
\begin{aligned}
& \partial_{t} \mathbf{u}=-(\mathbf{u} \cdot \nabla) \mathbf{u}+(\mathbf{b} \cdot \nabla) \mathbf{b}+\nu \nabla^{2} \mathbf{u}-\nabla p+\mathbf{f} \\
& \partial_{t} \mathbf{b}=-(\mathbf{u} \cdot \nabla) \mathbf{b}+(\mathbf{b} \cdot \nabla) \mathbf{u}+\eta \nabla^{2} \mathbf{b}
\end{aligned}
$$

where $\mathbf{u}=\mathbf{u}(\mathbf{x}, t)$ is the fluid velocity field and $p=p(\mathbf{x}, t)$ is the total, hydrodynamic + magnetic, pressure field divided by the constant mass density. The fluid viscosity $\nu$ and the magnetic diffusivity $\eta$ are taken to be equal, so that the magnetic Prandtl number $(\operatorname{Pr}=\nu / \eta)$ is unity. A known external force $\mathbf{f}=\mathbf{f}(\mathbf{x}, t)$ is used to maintain the system in a statistically stationary state in which the energy injected by the force is balanced on average by the energy loss due to dissipative effects. The MHD equations are completed by the incompressibility condition for the velocity flow field $(\nabla \cdot \mathbf{u}=0)$ and the divergence-free condition for the magnetic field $(\nabla \cdot \mathbf{b}=0)$. Because of the incompressibility condition, the pressure $p$ is a function of $\mathbf{u}$ and $\mathbf{b}$ that is solution of the Poisson equation obtained by applying the divergence operator to the equation 5 . In the MHD approximation, the self-consistent electric field $\mathbf{e}=\mathbf{e}(\mathbf{x}, t)$ is obtained from Ohm's law that can be expressed as follows:

$$
\mathbf{e}=-\mathbf{u} \times \mathbf{b}+\eta \nabla \times \mathbf{b} .
$$



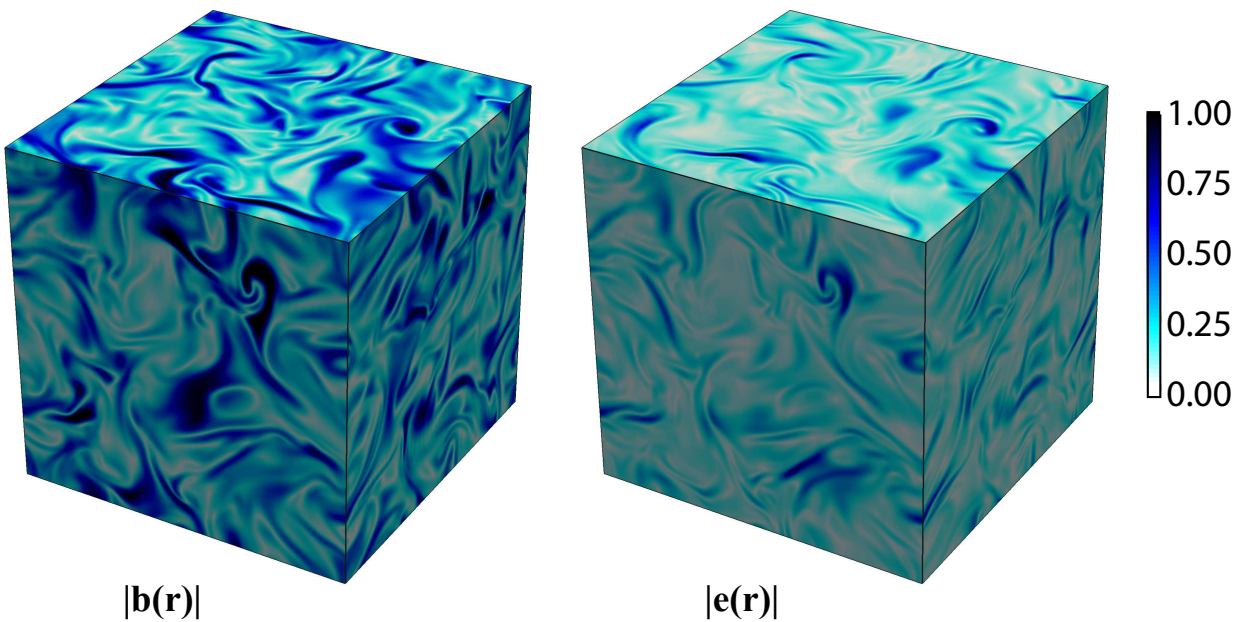

Figure 1: (Color online) Real space representation of the norm of the magnetic field (left) and the electric field (right) as solution of the MHD equations. Blue (dark grey) through green and yellow (light gray) depicts low to high values of the norms, respectively.

Well resolved solutions of the MHD equations have been obtained in a three dimensional cubic domain of size $\mathcal{L}^{3}$ with periodic boundary conditions using a pseudo-spectral solver $[14,15]$. The present study is designed to combine two objectives. First, it aims to study the transport of charged particles submitted to 'realistic' complex electric and magnetic fields with energy spectra and spatial correlations that are as close as possible to those of magneto-hydrodynamic turbulent fields. Second, the conservation of the total (electric potential + kinetic) particle energy is used to assess various numerical integration schemes. Strictly speaking, these two objectives cannot be fully conciliated. Indeed, the electric field generated by the nonlinear MHD equation is always composed of both an electric scalar potential and a magnetic vector potential $(\mathbf{b}=\nabla \times \mathbf{a})$ contributions:

$$
\mathbf{e}=-\nabla \phi-\partial_{t} \mathbf{a}
$$

The strategy adopted here is to simply remove the second term in the electric field and to retain only the $-\nabla \phi$ contribution (at a fixed time, more details follow in Section 3 ). Obviously, such an approximation implies that the electric field is not fully compatible with the nonlinear MHD equation. Nevertheless, it will still contain most of the twopoint correlation and spectral information while it will ensure that the total energy of the particles is conserved. In a sense, such an electric field should be much closer to realistic electrostatic turbulent field than randomly generated fields often used in the study of charged particle transport. In the present study, the conservation of the total energy for a charged particle is essential to clearly observe the numerical errors generated by the interplay of the field interpolation method with the particle trajectory solver.

In the MHD simulations, relatively modest grid sizes ( $128^{3}$ grid points) and moderate Reynolds numbers $R e \approx 88$ have been used. Despite the low Reynolds number value, the electric and the magnetic fields are charactised by rather complex structures as shown in FIG. 1, similar to the structures presented in [4]. Indeed, the objective of these type of 
studies is to investigate the influence of self-consistently computed electromagnetic fields on the diffusion of charged particles rather than achieving high resolution numerical simulation of MHD turbulence. The turbulent MHD fields are often characterised by the kinetic and magnetic energy spectra $E_{\mathbf{u}}(k)$ and $E_{\mathbf{b}}(k)$ as well as by the total energy spectrum $E_{\mathbf{t o t}}(k)=E_{\mathbf{u}}(k)+E_{\mathbf{b}}(k)$. A number of physically relevant quantities are directly derived from these quantities:

$$
\begin{aligned}
\mathcal{E}_{\mathbf{u}} & =\int d k E_{\mathbf{u}}(k) \\
\mathcal{E}_{\mathbf{b}} & =\int d k E_{\mathbf{b}}(k)
\end{aligned}
$$
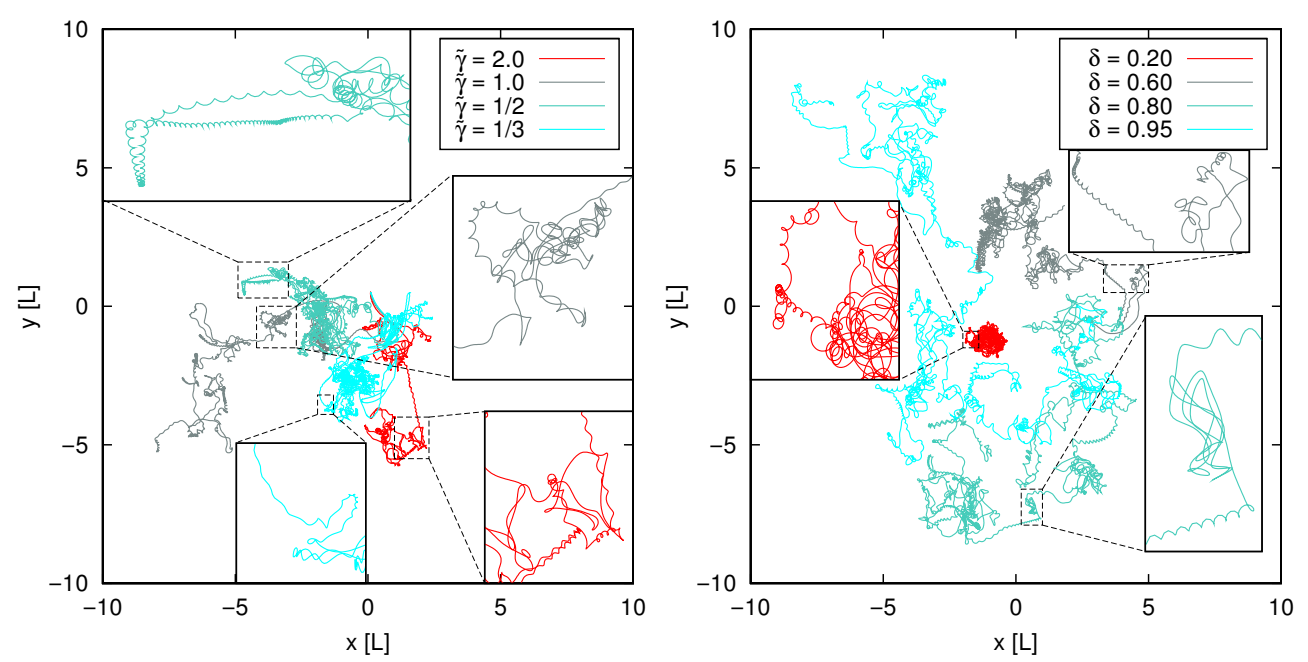

Figure 2: (Color online) Examples of trajectories with different $\gamma$ (left) and different energies (right).

$$
\begin{aligned}
\mathcal{D}_{\mathbf{u}} & =2 \nu \int d k k^{2} E_{\mathbf{u}}(k) \\
\mathcal{D}_{\mathbf{b}} & =2 \kappa \int d k k^{2} E_{\mathbf{b}}(k) \\
L & =\frac{1}{\mathcal{E}_{\text {tot }}} \int d k k^{-1} E_{\text {tot }}(k)
\end{aligned}
$$

where $\mathcal{E}_{\text {tot }}$ is the total energy given by the sum of the kinetic $\mathcal{E}_{\mathbf{u}}$ and the magnetic $\mathcal{E}_{\mathbf{b}}$ energies. The kinetic energy and the magnetic energy dissipations are noted respectively $\mathcal{D}_{\mathbf{u}}$ and $\mathcal{D}_{\mathbf{b}}$. The total dissipation in the system is then given by $\mathcal{D}_{\text {tot }}=\mathcal{D}_{\mathbf{u}}+\mathcal{D}_{\mathbf{b}}$. The integral length scale $L$ gives an estimate of the typical size of the largest structures in the MHD flow while $U=\sqrt{\mathcal{E}_{\mathbf{u}}}$ is the root mean square of the fluid velocity. The Reynolds number is then defined by:

$$
R e=\frac{U L}{\nu}
$$


It is also important to have an estimate of the typical size of the smallest structures in the MHD fields. Since the magnetic Prandtl number is equal to 1, the Kolmogorov length usually introduced in Navier-Stokes turbulence for non conductive fluids should provide a good estimate of the smallest structure size:

$$
\ell_{\nu}=\left(\frac{\nu^{3}}{\mathcal{D}_{\text {tot }}}\right)^{1 / 4}
$$

The ratio

$$
\gamma=\frac{\ell}{\ell_{\nu}}
$$

is thus a dimensionless parameter that indicates how much the particle trajectories are influenced by the spatial fluctuations of the MHD fields. For very small $\gamma$, the particles will locally experience constant electric and magnetic fields and should follow helical trajectories corrected by the $\mathbf{e} \times \mathbf{b}$ drift. For very large $\gamma$, the particles do not really interact with the MHD turbulent structures and the Lorentz force acts almost like a random force. The numerical tests presented in the following section are focused on intermediate values of $\gamma$, slightly smaller than 1 in which the helical motion of the particle can still be detected although it is strongly affected by the electromagnetic field inhomogeneities.

The ratio $\ell / L$ may also play a role in the dynamics of the particles. Indeed $L$ can also be interpreted as a correlation length of the turbulent field and if the ratio $\ell / L$ is of order unity, the particle trajectories might be influenced by finite correlation length effects. In the following, $\ell / L$ will however be systematically be a very small number (less than 0.02).

Two parameters control the global behavior of the particles: the coupling parameter $\gamma$ and the total energy of the particle $E$. The parameter $\gamma$ directly affects the amplitude of the force acting on the particle. The total energy fixes a five-dimensional manifold that contains the trajectory of the particle. In the following study, particles with different energies and different charge to mass ratios, i.e. different $\gamma$ are studied. Since these various populations of particles have different characteristic time scales, the time normalisation will be systematically done with respect to a timescale of the fields: the large eddy turnover time of MHD: $\tau_{\mathrm{mhd}}=L / U$.

A few example trajectories are shown in FIG. 2. Low energy particles are simply trapped. As particle energies increase, trajectories can exist in more and more small volumes around the minima of the electric potential. For sufficient energy, trapping and flight regimes alternate, with the intervals of particle flight tending to be adiabatic (i.e. the magnetic moment can be conserved during these intervals).

\section{Numerical schemes}

To integrate particle trajectories, the splitting technique described in [8] is used, together with a 4th order composition method described in [16], with a constant timestep. Indeed, geometric integration techniques such as the splitting-composition methods requires a constant timestep [6] in order to preserve conservation properties. In a previous work [12], a hierarchy of spline interpolations for dynamic fields represented on regular, 
rectangular spatial grids has been presented. This approach is briefly summarized below in order to introduce the notations. Given a function $f$, the polynomial

$$
s^{(n)}(x)=\sum_{k=0}^{n} a_{k}^{(n)} x^{k}
$$

is called an order $n$ Hermite spline interpolation of $f$ for $x \in[0,1]$ if it satisfies the conditions

$$
\left\{\begin{array}{l}
\frac{d^{l} s^{(n)}}{d x^{l}}(0)=\frac{d^{l} f}{d x^{l}}(0) \\
\frac{d^{l} s^{(n)}}{d x^{l}}(1)=\frac{d^{l} f}{d x^{l}}(1)
\end{array}, l=0 \ldots m\right.
$$

where $m=(n-1) / 2$ is the highest derivative that is kept continuous by the interpolation $s$. Here, the derivatives are approximated by their centered difference approximations using $2 g+1$ grid points:

$$
\frac{d^{l} f}{d x^{l}}(j) \approx f^{\langle l, q\rangle}(j)=\sum_{i=j-g}^{j+g} c_{i}^{(l, q)} f(i),
$$

Finite differences are used because, for 3D fields, the cost of keeping all the additional derivatives in the computer's memory becomes prohibitive. With centered differences, the solution of (18) can be rewritten as

$$
s^{(n, q)}(x)=\sum_{i=0-g}^{1+g} f(i) \beta_{i}^{(n, q)}(x) .
$$

where $q=2 g+2$ is the number of grid nodes contributing to the approximation. The splines are then controlled by two parameters: the order $n$ of the polynomial or the smoothness $m$ and $q$ the order of the centered differences scheme which is related to the accuracy. It is important to note that the computational cost of the method increases rapidly with $q$. With this method, 13th order splines can be computed using 8 grid points, requiring no extra memory for the derivatives. Tensor product splines can be used for 3D fields:

$$
s^{(n, q)}(\mathbf{r})=\sum_{0-g}^{1+g}\left(f(\mathbf{i}) \prod_{j=1}^{3} \beta_{i_{j}}^{(n, q)}\left(x_{j}\right)\right) \equiv S^{(n, q)}(\mathbf{r}) f
$$

The operator $S^{(n, q)}$ can be used in two ways [10] for the magnetic field $\mathbf{b}$ :

$$
\hat{\mathbf{b}}^{(n, q)}=S^{(n, q)} \mathbf{b} \text { or } \tilde{\mathbf{b}}^{(n, q)}=\nabla \times\left(S^{(n, q)} \mathbf{a}\right)
$$

Similarly we consider $\hat{\mathbf{e}}^{(n, q)}=S^{(n, q)} \mathbf{e}$ or $\tilde{\mathbf{e}}^{(n, q)}=-\nabla\left(S^{(n, q)} \phi\right)$. Specifically, $\phi$ is computed in Fourier space from the MHD fields, and then the real space representations of both $\phi$ and $-\nabla \phi$ are computed (so the fields are known on the grid nodes of the real space grid). It is this real space representation of $-\nabla \phi$ that is noted $\mathbf{e}$ in the above formula for $\hat{\mathbf{e}}$. Note that $\tilde{\mathbf{b}}$ is exactly divergence free by construction, and $\tilde{\mathbf{e}}$ is exactly curl free. Ideally, the total energy of the particle should be preserved, but errors appear when $\hat{\mathbf{e}}$ is used because $\hat{\mathbf{e}}$ is not a conservative field exactly (as opposed to $\tilde{\mathbf{e}}$ ). The qualitative differences between the two resulting hierarchies of Lorentz forces $\left(\hat{\mathbf{F}}^{(n, q)}\right.$ and $\left.\tilde{\mathbf{F}}^{(n, q)}\right)$ are discussed in the following section. 


\section{Results}

\subsection{Energy conservation}

In the following, we will consider an ensemble of $N$ particles evolving according to the equations 2. The six-dimensional phase space coordinates of each particle $(i=1, \ldots, N)$ will be denoted $\xi^{i}(t)=\left(\mathbf{r}^{i}(t), \mathbf{v}^{i}(t)\right)$. In a frozen electromagnetic field the total energy of each particle

$$
H\left(\xi^{i}(t)\right)=\frac{m}{2} \mathbf{v}^{i}(t)^{2}+q \phi\left(\mathbf{r}^{i}(t)\right)
$$

is conserved. This energy conservation is expressed by $H\left(\xi^{i}(t)\right)=E_{0}^{i}$, where $E_{0}^{i}$ is the initial value of the total energy of the $i$ th particle. Such a relation defines a fivedimensional manifold in the six-dimensional phase space of the particle. Without loss of generality, the charge will be assumed to be positive, so that the peak of the electric potential $\phi_{\max }$ corresponds to the largest possible value of the particle potential energy. The minimal value of the electric potential $\phi_{\min }$ can then be associated to the minimal value of the energy of a particle $E_{0}>q \phi_{\min }$. The properties of the trajectory will depend quite strongly on the positive parameter

$$
\delta^{i}=\frac{E_{0}^{i}-q \phi_{\min }}{q\left(\phi_{\max }-\phi_{\min }\right)}
$$

When the initial energy of the particle $E_{0}^{i}$ is lower than $q \phi_{\max }\left(\delta^{i}<1\right)$, the particle can only access a sub-region of the physical space $\Omega\left(\delta^{i}\right)$ defined by $\Omega\left(\delta^{i}\right)=\left\{\mathbf{r} \mid q \phi(\mathbf{r})<E_{0}^{i}\right\}$. The domain $\Omega\left(\delta^{i}\right)$ may be unbounded and exhibit a labyrinthine structure. However, it may also be bounded, in which case the particle is trapped. Obviously, the number of trapped particles is expected to increase for smaller $\delta^{i}$. In the following, the numerical tests will be entirely focused on population of particles with $0<\delta^{i}<1$.

As mentioned earlier, a value of $\gamma$ slightly smaller than 1 corresponds to particle trajectories that are simultaneously subject to a space varying electromagnetic field while they still exhibit a helical motion in certain regions of intense magnetic field. Combining all the parameters that enter $\gamma$, a reference value $\gamma_{0} \approx 0.2$ has been chosen. In the following, results will be presented for various values of $\tilde{\gamma}=\gamma / \gamma_{0}(\tilde{\gamma}=1 / 3,1 / 2,1,2)$. It is also useful to introduce an energy scale given by $\varepsilon_{0}=q \phi_{\max }$ which corresponds to the energy of a particle characterized by $\delta=1$.

All the tests are performed with a population of $N$ particles characterised by the same $\delta$. Although the trajectory $\xi^{i}(t)$ should only depend on its initial condition $\xi^{i}(0)$ and on the parameter $\gamma$, in practice, in a numerical integration, $\xi^{i}(t)$ also depends on the timestep $\tau$ as well as on the interpolation scheme used to estimate the electromagnetic field at the particle position. It has been verified however that, for high order interpolation schemes, the expected accuracy of the integration scheme is preserved for both $\hat{\mathbf{F}}^{(n, q)}$ and $\tilde{\mathbf{F}}^{(n, q)}$ estimates for the Lorentz force. A fourth order time integration scheme was used, and the integration error is proportional to the fourth power of the time-step $\left(\tau^{4}\right)$. This extends the observations reported in [12] to complex turbulent MHD fields. It should be noted however that the $\tau^{4}$ scaling is lost when the time-steps are too large. In that case, the Taylor expansion used to derive the numerical scheme may indeed lose its convergence property. The $\tau^{4}$ scaling is also lost when the time steps are too small since the rounding errors become dominant. 
The analysis of the energy conservation shows more differences between the estimates $\hat{\mathbf{F}}^{(n, q)}$ and $\tilde{\mathbf{F}}^{(n, q)}$. The quantity

$$
\varepsilon(t ; \tau)=\frac{1}{N} \sum_{i=1}^{N}\left|H\left(\xi^{i}(t ; \tau)\right)-E_{0}^{i}\right|
$$

gives an estimate of the average error generated by the numerical integration of the trajectory on the total energy of the particle. Not surprisingly, the schemes $\tilde{\mathbf{F}}^{(n, q)}$ for which the interpolation of the magnetic field is exactly divergence free and the interpolation of the electric field is exactly curl free appear to be much more performant in conserving the energy. For instance, FIG. 3 shows a clear difference between $\hat{\mathbf{F}}^{(7,6)}$ and $\tilde{\mathbf{F}}^{(7,6)}$. First, the value of $\varepsilon(t ; \tau)$ is much larger for $\hat{\mathbf{F}}^{(7,6)}$. Second, the value of $\varepsilon(t ; \tau)$ appears to be independent of $\tau$ for $\hat{\mathbf{F}}^{(7,6)}$ while it decreases significantly with $\tau$ for $\tilde{\mathbf{F}}^{(7,6)}$. Such a property indicates that the energy error in the integration of the particle trajectory is entirely dominated by the interpolation scheme when the class of interpolation $\hat{\mathbf{F}}^{(n, q)}$ is used, while, on the contrary, it is dominated by the time integration scheme when the class of interpolation $\tilde{\mathbf{F}}^{(n, q)}$ is used.
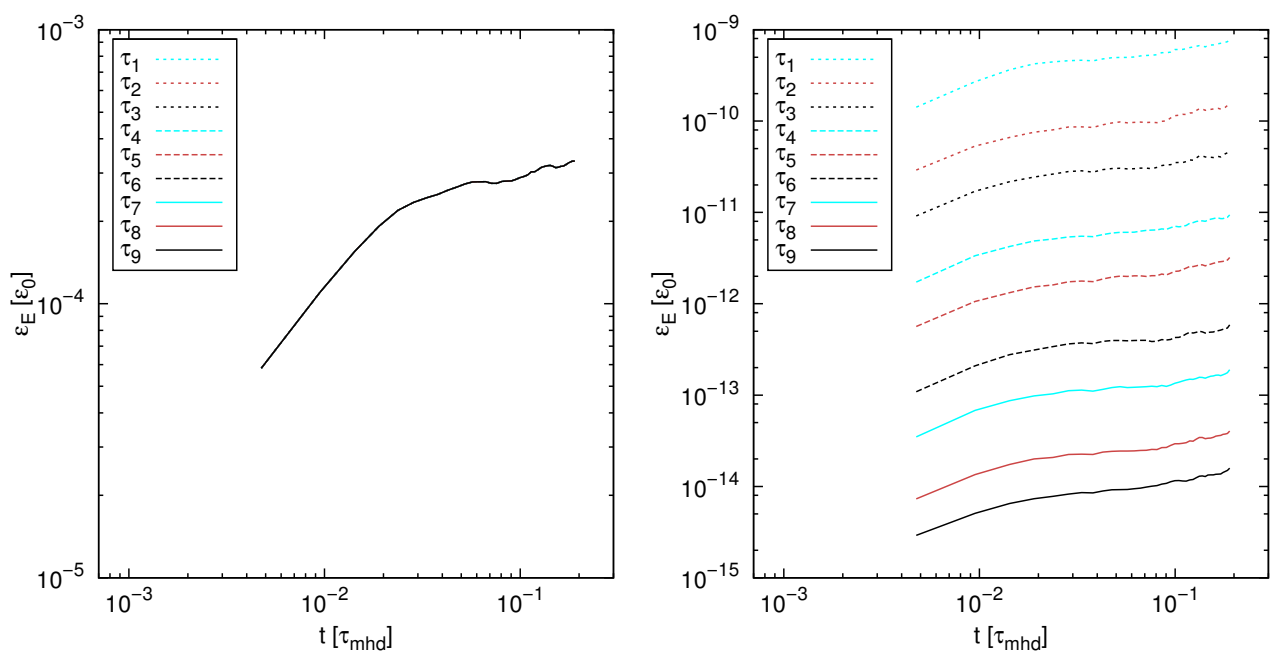

Figure 3: (Color online) Evolution of energy error, for several timesteps, $\hat{\mathbf{F}}^{(7,6)}$ left and $\tilde{\mathbf{F}}^{(7,6)}$ right The time-steps are constructed from $\tau_{0} \approx 9.5 \cdot 10^{-3} \tau_{\mathrm{mhd}}: \tau_{1}=\tau_{0} / 2, \tau_{2}=\tau_{0} / 3, \tau_{3}=\tau_{1} / 2, \tau_{4}=\tau_{2} / 2$, ... Note that all the curves overlap in the $\hat{\mathbf{F}}^{(7,6)}$ case. The particles are characterised by $\tilde{\gamma}=1$ and $\delta=0.5$.

\subsection{Interpolation technique}

The second test concerns the dependency of the energy error on the type of interpolation used. The error is obviously expected to decrease for improved interpolation scheme. However, it is also very interesting to quantify how this error decreases. For this test, the total time of the simulation is set to $T_{0}=20 \tau_{0}$ (with $\tau_{0} \approx 9.5 \cdot 10^{-3} \tau_{\text {mhd }}$ ), and the time step $\tau_{5}=2^{-3} \tau_{0}$ is used.

In FIG. 4, a qualitative difference between the two types of interpolations, $\hat{\mathbf{F}}$ and $\tilde{\mathbf{F}}$ is again observed. For the direct interpolation $\hat{\mathbf{F}}$, the energy error depends mostly on the 

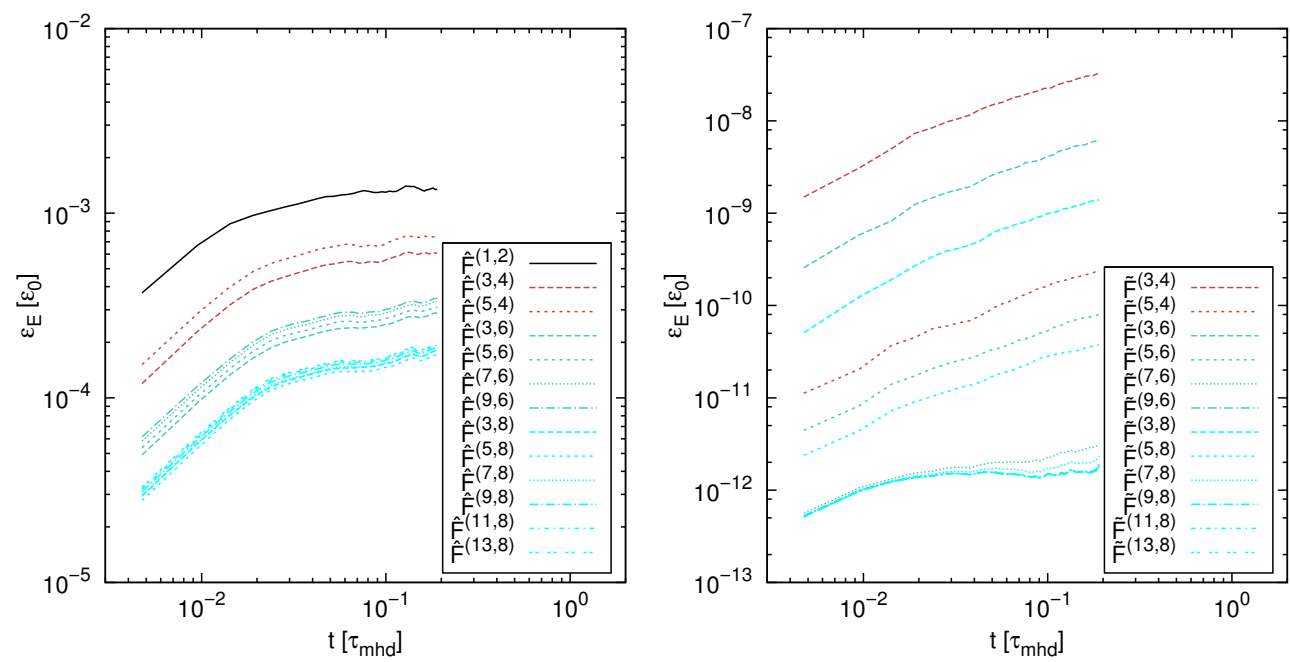

Figure 4: (Color online) Evolution of energy error for several $\hat{\mathbf{F}}(n, q)$ (left) and $\tilde{\mathbf{F}}(n, q)$ (right).

number of grid points $q$ used for the interpolation and not significantly on $n$. However, increasing $q$ does not decrease substantially the error, while the number of computations needed for one interpolation is proportional to $q^{3}$. In the second class of interpolation schemes $\tilde{\mathbf{F}}$, the energy error depends much more strongly on the interpolation smoothness $n$ than on the number of points $q$.

\subsection{Statistical analysis of trajectories}

Two sets of longer runs have been performed, with varying physical parameters $\gamma$ and $\delta$. In the first set, $\delta=0.5$ is fixed and $\tilde{\gamma}$ varies $(\tilde{\gamma} \in\{2,1,1 / 2,1 / 3\})$. In the second set, $\tilde{\gamma}=2 / 3$ is fixed and $\delta$ varies $(\delta \in\{0.05,0.2,0.4,0.6,0.8,0.95\}) . N=1000$ trajectories are integrated, initialized as before with random positions and fixed total energy. The total integration time is $T \approx 95 \tau_{\mathrm{mhd}}$. For each set of runs, the time step is adapted so that the local integration errors are at a fixed level. Obviously, the time step is thus a function of the scheme as well as of the parameters $\delta$ and $\tilde{\gamma}$. FIG. 5 shows how the time-step can be estimated from the error $\varepsilon(t ; \tau)$ (local integration error for the trajectory itself, see [12] for detailed methodology). Both sets of runs have been performed for the five interpolation schemes $\hat{\mathbf{F}}^{(1,2)}, \hat{\mathbf{F}}^{(3,4)}, \hat{\mathbf{F}}^{(7,6)}, \tilde{\mathbf{F}}^{(3,4)}$ and $\tilde{\mathbf{F}}^{(7,6)}$. Due to the fact that the effective order of time integration solver is 2 for linear interpolation and for $\tilde{\mathbf{F}}^{(3,4)}$, a second order solver was used for the linear and cubic interpolations, and a larger local integration error was allowed for these simulations.

Various diagnostics are computed for each set of trajectories: the mean squared displacement, the probability density function (PDF) of the velocity and the PDF of the displacement corresponding to a fixed time interval. All PDFs are computed as simple histograms. The average energy error is also computed, as a measure of the accuracy of each individual simulation. 

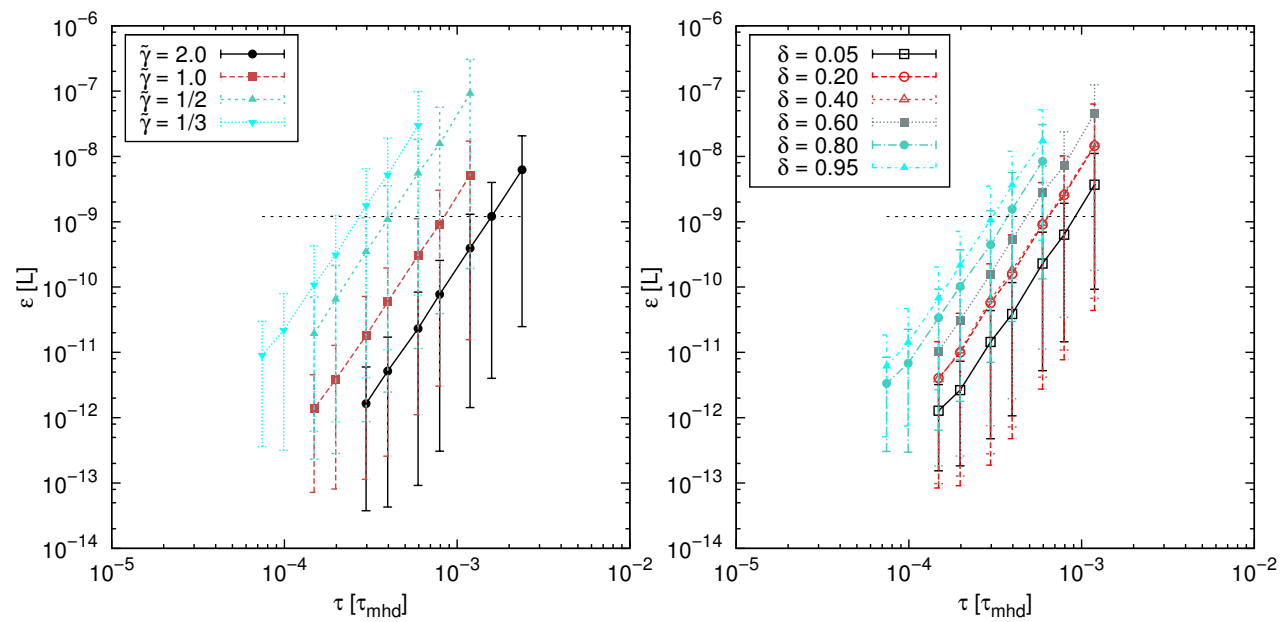

Figure 5: (Color online) Local integration errors for various physical parameters and $\tilde{\mathbf{F}}^{(7,6)}$. The horizontal dashed lines represent the error level that was imposed for the simulations. The time step is estimated by considering the value of $\tau$ that corresponds to the intersection of this horizontal line with the error curves.

\subsubsection{Influence of the coupling parameter}

The influence of the coupling parameter $(\tilde{\gamma})$ on the average error on the energy is shown in FIG. 6. Clearly, the error appears to be almost proportional to $\tilde{\gamma}$ for the class of interpolation $\hat{\mathbf{F}}$. The curves corresponding to $\hat{\mathbf{F}}^{(3,4)}$ are not shown for clarity but they would be halfway between the $\hat{\mathbf{F}}^{(1,2)}$ and $\hat{\mathbf{F}}^{(7,6)}$ curves. On the contrary, the average error on the energy is almost independent of $\tilde{\gamma}$ for the class of interpolation $\tilde{\mathbf{F}}$. Again, the error is clearly smaller for $\tilde{\mathbf{F}}^{(7,6)}$ than for $\hat{\mathbf{F}}^{(7,6)}$, and while the numerical error is strongly correlated with the physical parameter $\tilde{\gamma}$ for the direct interpolation, this correlation is no longer present for the $\tilde{\mathbf{F}}$ interpolation.

The distance between the initial position of the particle $i$ and its position at time $t$ is noted $\sigma_{i}(t)=\left|\mathbf{r}_{i}(t)-\mathbf{r}_{i}(0)\right|$ and will be referred to as the displacement of particle $i$. The mean square displacement of the particle population is then given by $\sigma(t)^{2}=\left\langle\sigma_{i}(t)^{2}\right\rangle$, which is shown in FIG. 7. Also, the velocity distribution of the particles is displayed FIG. 8. In these figures, the continuous line corresponds to the linear interpolation and the other interpolations are represented with variations of dashed lines. It appears that the mean square displacement and the probability distribution of the velocity are almost unaffected by the type of interpolation. Neither the class nor the order seem to significantly modify the results and the curves corresponding to the same $\tilde{\gamma}$ overlap almost perfectly.

In FIG. 7 (the left graph), the time evolution of the average mean squared displacement is shown for varying $\tilde{\gamma}$. A ballistic regime $(\sigma(t) \propto t)$ is observed for small times. Asymptotically, a subdiffusive regime $\left(\sigma(t) \propto t^{3 / 8}\right)$ seems to be observed for all the values of $\tilde{\gamma}$ chosen for this study. Not surprisingly, both the PDF of $\sigma$ and of the velocity are more and more spread for smaller and smaller $\tilde{\gamma}$, which correspond to larger and larger coupling constants. 

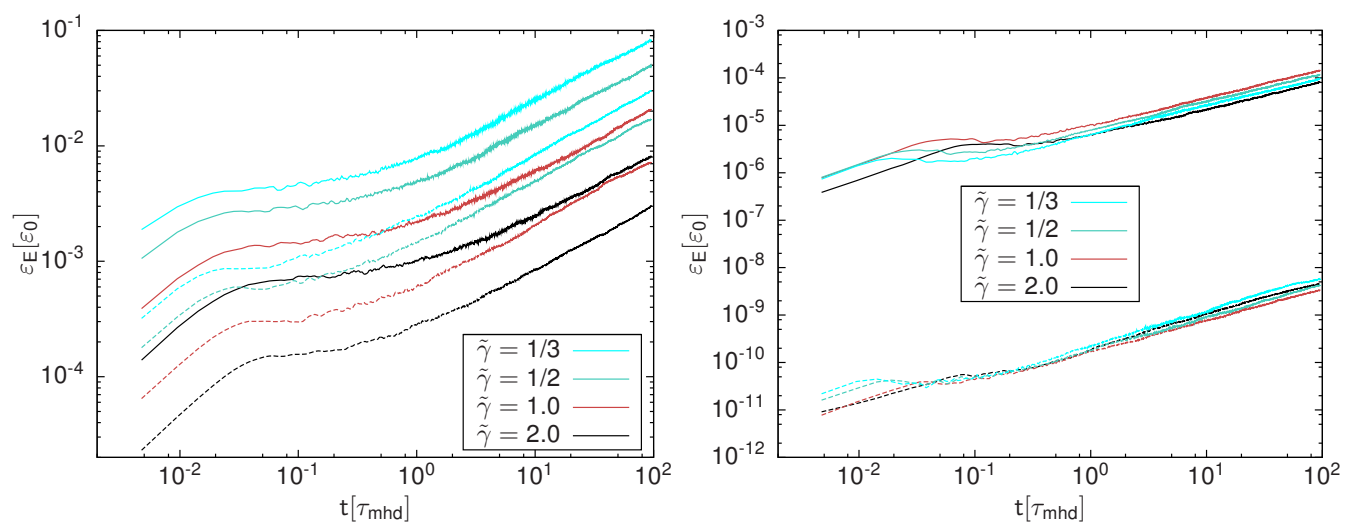

Figure 6: (Color online) Energy error for various $\tilde{\gamma}$. Left: energy errors for $\hat{\mathbf{F}}^{(1,2)}$ (continuous line) and $\hat{\mathbf{F}}^{(7,6)}$ (dashed line). Right: energy errors for $\tilde{\mathbf{F}}^{(3,4)}$ (continuous line) and $\tilde{\mathbf{F}}^{(7,6)}$ (dashed line). Errors for the same $\tilde{\gamma}$ are shown with the same color (shade of gray).

\subsubsection{Influence of the particle energy}

The interplay between the particle energy and the interpolation method has also been analyzed using the same diagnostics. First, the average error on the energy is analysed in FIG. 9. There seems to be a correlation between $\delta$ and the value of the energy errors for the direct interpolations $\hat{\mathbf{F}}$, while the same is not true for the second class of fields $\tilde{\mathbf{F}}$.

The graph on the right of FIG. 7 shows the influence of $\delta$ on the mean square displacement, and this influence on the PDFs of the displacement and of the velocity is shown in FIG. 10. Again, the continuous line corresponds to linear interpolation and the other interpolations are represented with variations of dashed lines. It appears that mean square displacement is not really affected by the order of the interpolation scheme and all curves collapse. Unlike the influence of $\tilde{\gamma}$, the influence of $\delta$ on $\sigma^{2}(t)$ is very strong. Again, for small times, a ballistic regime is observed for all values of $\delta$. However, the asymptotic regime varies between a classical diffusion regime for large $\delta$ to a fully trapping regime for small $\delta\left(\sigma(t) \propto t^{0}\right)$. In between, various anomalous diffusion regimes are observed with $\left(\sigma \propto t^{\alpha}\right)$ with $0<\alpha<1 / 2$. This strong influence was expected, because $\delta$ is directly related to the volume of physical space that the particles can visit. Not surprisingly, the PDF of the displacement is more spread for increasing particle energy as shown in the middle part of FIG. 10.

The effect of the interpolation scheme is only visible on the PDF of the velocity for small $\delta$. The displacement and velocity PDFs for $\delta=0.05$ are shown in more detail in FIG. 11. In particular, the linear interpolation fails to show the presence of two separate particle populations and both direct interpolations seem to smooth the secondary maximum in the velocity PDF. In practice, no matter what kind of $\hat{\mathbf{e}}$ interpolation is used (i.e. linear or some spline), some inexact electrostatic potential can be inferred for this electric field - it is inexact because $\hat{\mathbf{e}}$ can not be an exact divergence. Allowing for the use of this inexact potential, we can go on to note that its "isosurfaces" can be different from the isosurfaces of $\hat{\phi}$. There are then two reasons for the discrepancies: firstly, the volumes that can be visited by particles have different shapes for different interpolations; secondly, the volumes that can be visited by particles moving under the influence of $\hat{\mathbf{e}}$ 

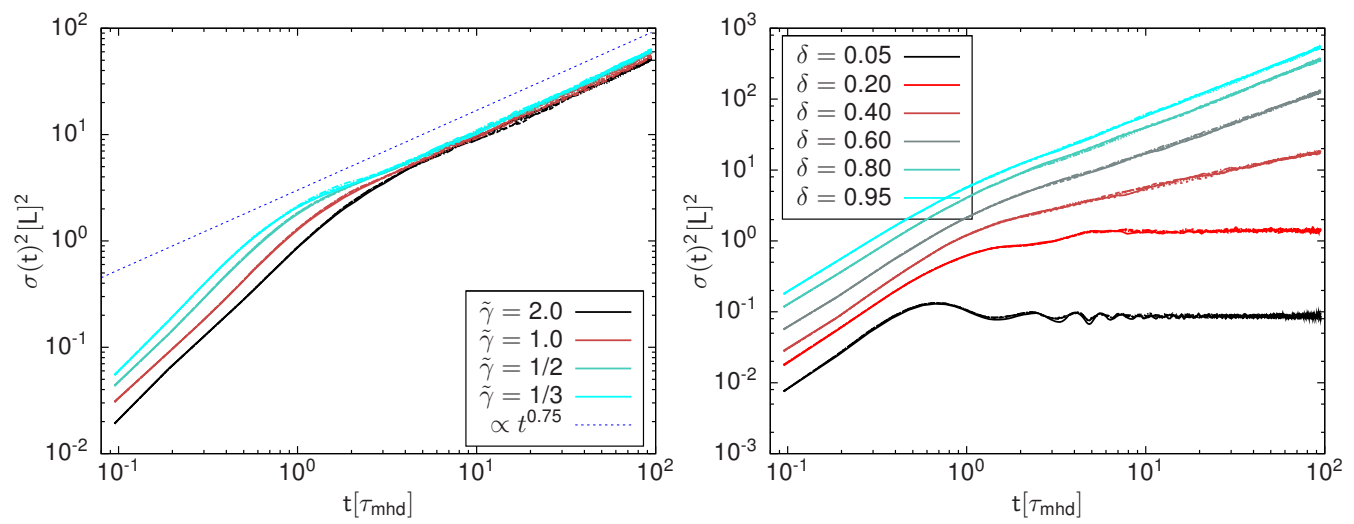

Figure 7: (Color online) Mean squared displacement as a function of time for varying $\tilde{\gamma}$ (left) and varying $\delta$ (right). The curves for $\hat{\mathbf{F}}^{(1,2)}, \hat{\mathbf{F}}^{(3,4)}, \hat{\mathbf{F}}^{(7,6)}, \tilde{\mathbf{F}}^{(3,4)}$ and $\tilde{\mathbf{F}}^{(7,6)}$ overlap for the same $\tilde{\gamma}$ and are shown using the same color (shade of grey) for reference.
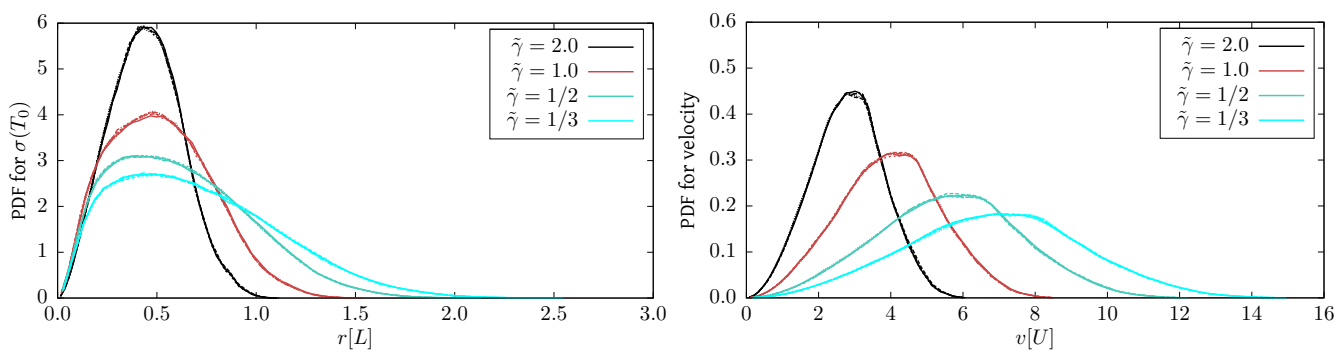

Figure 8: (Color online) Displacement PDF (left) and velocity PDF (right) for varying $\tilde{\gamma}$. The curves for $\hat{\mathbf{F}}^{(1,2)}, \hat{\mathbf{F}}^{(3,4)}, \hat{\mathbf{F}}^{(7,6)}, \tilde{\mathbf{F}}^{(3,4)}$ and $\tilde{\mathbf{F}}^{(7,6)}$ overlap for the same $\tilde{\gamma}$ and are shown using the same color (shade of grey) for reference.

are not well-defined, thus even though for short time-spans the results might be close, the overall statistics (that allow the observation of separate particle populations) will start to diverge as errors due to the nonconservative nature of $\hat{\mathbf{e}}$ have time to grow. It is likely that, if finer grids are used (i.e. the fields are taken from simulations where the grid constant is smaller relative to the dissipation length), the discrepancies will be less pronounced (in extreme cases even for $\hat{\mathbf{e}}^{(1,2)}$ ), at least if the same time-spans are being used. However, such a finer grid would imply a higher cost of the MHD simulations, which seems unnecessary given that the better $\tilde{\mathbf{e}}^{(7,6)}$ are available.

\section{The case of time-varying fields}

Although this study is not intended to be an in-depth analysis of particle transport by MHD turbulence, this section mentions briefly the case of transport by time-evolving MHD turbulence. Charged particles can be strongly accelerated by MHD turbulence [4], thus it can become quite expensive to study their motion - larger energies imply smaller characteristic time-scales. In this preliminary example, a term corresponding to Stokes 

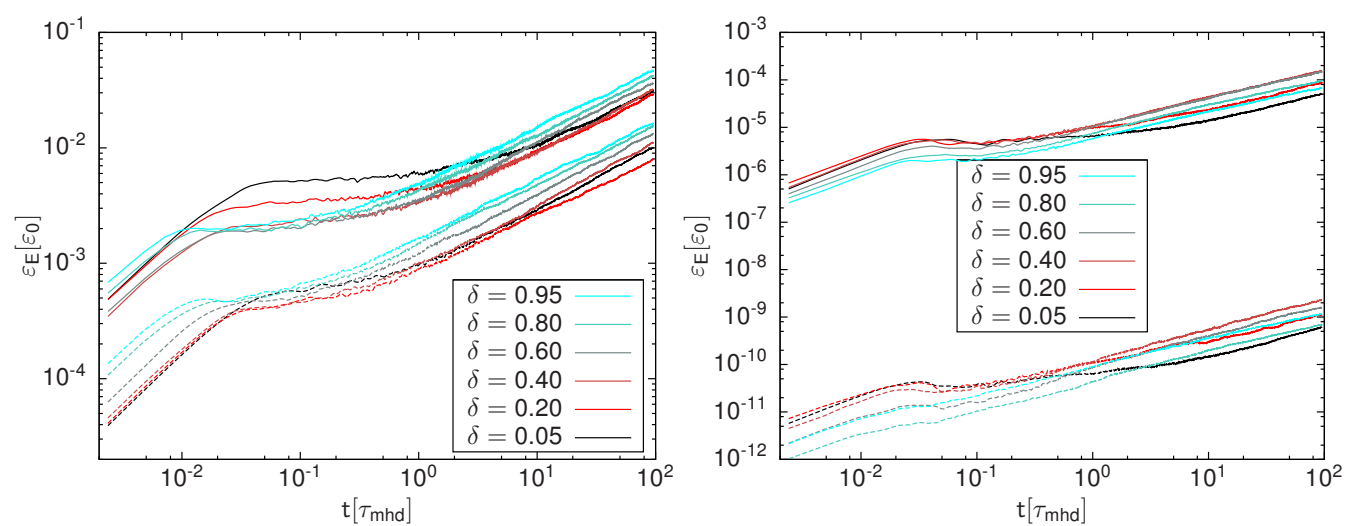

Figure 9: (Color online) Energy error for varying $\delta$. Results for $\hat{\mathbf{F}}^{(1,2)}$ (continuous line) and $\hat{\mathbf{F}}^{(7,6)}$ (dashed line) are shown in the top figure, and for $\tilde{\mathbf{F}}^{(3,4)}$ (continuous line) and $\tilde{\mathbf{F}}^{(7,6)}$ (dashed line) in the bottom.
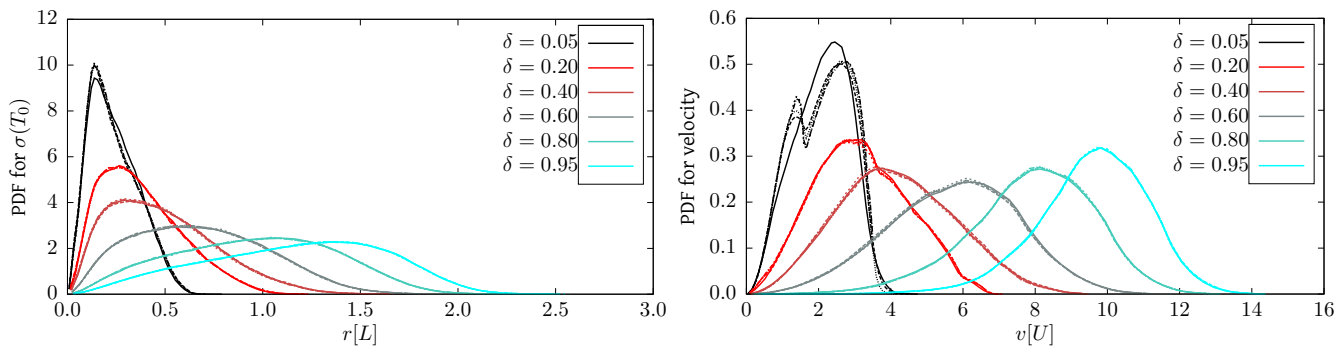

Figure 10: (Color online) Displacement PDF (left) and velocity PDF (right) for varying $\delta$. The curves for $\hat{\mathbf{F}}^{(1,2)}, \hat{\mathbf{F}}^{(3,4)}, \hat{\mathbf{F}}^{(7,6)}, \tilde{\mathbf{F}}^{(3,4)}$ and $\tilde{\mathbf{F}}^{(7,6)}$ overlap for the same $\delta$ and are shown using the same color (shade of grey) for reference. The case $\delta=0.05$ is expanded for clarity in FIG. 11.

drag is added to the Lorentz force in the particle equation:

$$
\begin{aligned}
\partial_{t} \mathbf{r} & =\mathbf{v} \\
\partial_{t} \mathbf{v} & =\frac{1}{\ell}(\mathbf{e}(\mathbf{r})+\mathbf{v} \times \mathbf{b}(\mathbf{r}))+\frac{1}{\tau}(\mathbf{u}(\mathbf{r})-\mathbf{v})
\end{aligned}
$$

This term models "collisions", characterized by a frequency $\tau^{-1}$, between a charged test particle and the components of its medium - the electrically conducting fluid (i.e. plasma) for which the MHD equations are solved. Physically, the electromagnetic fields obtained from the MHD formalism can be considered as the large wavelength, low frequency component of the "true" electromagnetic fields obtained from a full kinetic theory description. For a plasma medium, the collisions occuring between its constituents are already considered in the fluid approximation due to the presence of viscosity. However, the collisions between the charged test particle and the plasma constituents still need to be modelled. Considering massive test particles (impurities), multiple collisions with the plasma constituents will lead to an effective drag force generated by the fluid on the test particle. Note that it is assumed these impurities are insufficient to influence the MHD flow itself, an appropriate assumption when one considers a limited number of test 

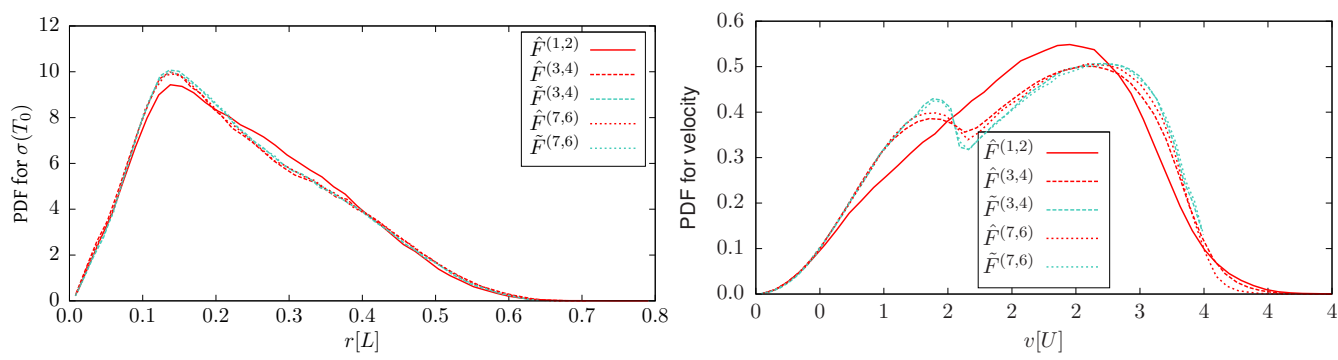

Figure 11: Displacement PDF (left) and velocity PDF (right) for $\delta=0.05$.

particles. Adding a drag force is important from a numerical point of view since, without it, a fraction of the particles may experience resonance phenomena and gain energy indefinitely in a time-evolving field. The ratio of characteristic times defines the Stokes number:

$$
S t=\frac{\tau}{\tau_{\mathrm{mhd}}}
$$

Simulations were run with Re varying mostly between 100 and 110, and six particle species: two values of $\gamma$ (approximately 0.46 and 0.70 ) and three values of St (approximately 2, 15 and 150) - obviously, all these values evolved throughout the simulation. $6^{3}$ particles were initialized on a regular spatial grid, with initial velocities equal to the local fluid velocity $\mathbf{u}$.
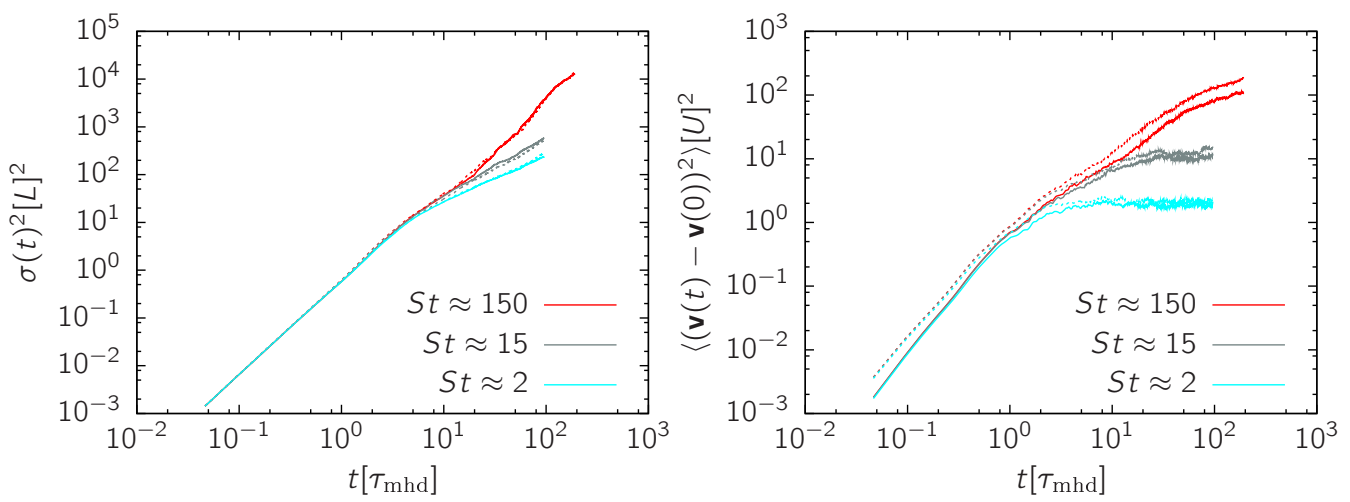

Figure 12: Mean squard displacements for position and velocity, for different values of the $S t$ number. Continuos lines denote $\gamma \approx 0.70$ and dotted lines $\gamma \approx 0.46$.

Figure 12 clearly shows that, as drag is decreased, particles have the tendency to be accelerated for very long times. Large values of the $\gamma$ parameters were used here, and they only influence the initial acceleration of the particles, the long time behavior being dominated by the $S t$ number. This can be deduced from the velocity displacement, where the curves with the same $\gamma$ collapse for the initial ballistic phase. On the contrary, in the assymptotic regime, these curves collapse for the same $S t$ number. It is interesting that the transport regime is superdiffusive for the $S t \approx 15$ case (the exponent is around 1.2), even though their kinetic energy reaches a quasistationary regime. Normal diffusion is 
observed for the $S t \approx 2$ case, and it is not clear if the asymptotic regime is reached for the $S t \approx 150$ case.
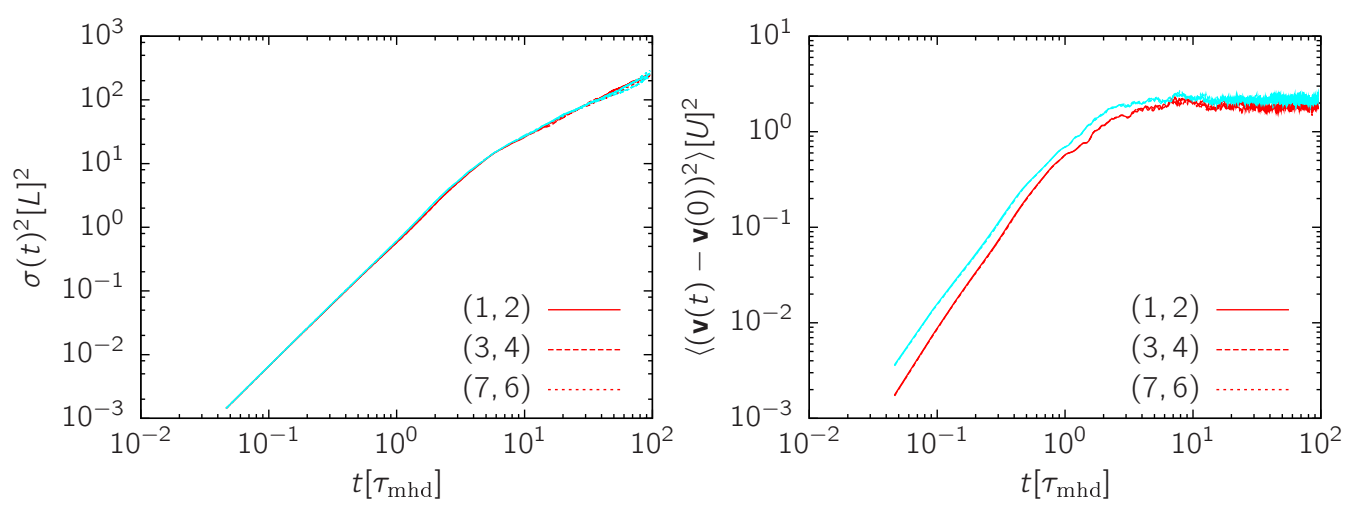

Figure 13: Mean squard displacements for position and velocity, for different interpolations $(S t \approx 2)$. Red (dark grey) lines denote $\gamma \approx 0.70$ and cyan (light gray) lines $\gamma \approx 0.46$.

Because a drag term is added to the Lorentz force, it was expected that the interpolation scheme would play a less important role. Figure 13 shows how for the $S t \approx 2$ value the interpolation scheme does not affect transport in position or velocity space. Even though the curves in velocity space do diverge, they have the same statistical properties. Similar behaviors are observed for the other values of St. In future studies, lower values of $\gamma$ will be investigated.

\section{Conclusions and discussion}

A number of numerical schemes have been developed and tested for tracking charged particle trajectories in a complex electromagnetic field. In particular, the performances of these schemes are evaluated in terms of energy conservation in the case of stationary electromagnetic fields, obtained by removing the solenoidal part of the self-consistent electric field obtained from direct numerical simulations of MHD turbulence. Clearly, interpolation methods that preserve the topological properties of the fields (exactly divergence free magnetic field and curl free electric field) appear to be much more efficient in conserving the energy, and there are qualitative differences between the two classes of approximations described. The interpolation schemes seem to have little influence on the PDF of the particle displacement and on the PDF of the particle velocity, even when they are characterized by rather poor performances in conserving the energy. Hence, in many cases, energy conservation does not seem to be crucial in characterizing the basic properties of the transport of particles. This is most likely due to the fact that small interpolation errors do not directly influence the specific field properties which characterize the transport regime for a large ensemble of particles with sufficient total energy.

However, the results obtained for low energy particles show that this conclusion cannot be fully general. Indeed, when a significant fraction of the particles are trapped, the PDF of the velocity is not properly reproduced by low order interpolation schemes. It seems thus preferable to use high order interpolation scheme if the transport properties 
are expected to deviate from standard diffusion, such as the sub-diffusive regimes observed for particle population with low $\delta$. Moreover, it appears clearly that the statistics of the velocity are affected more by the interpolation scheme than the statistics of the position (displacement). It is thus anticipated that the statistics of the acceleration and of higher order derivatives are even more sensitive to the interpolation method.

A brief investigation of the general case seems to imply that, in the case of particles experiencing Stokes drag, the interpolation scheme does not play an important role. Indeed, it can be the case that interpolation errors are hidden by the effects of the drag force, but it is not clear in which situations this is true. Even more so, it is not clear what would happen in other models where turbulent, discretized fields are being used, such as PIC codes. For such different problems, we expect that the interpolation scheme will indeed play an important role (especially since in some PIC codes energy conservation for the full system is desired).

\subsection{Acknowledgements}

We would like to thank B. Weyssow for his helpful comments. This work has been partly supported by the contract of association EURATOM-Belgian state. D.C. and C.C.L. are participants in the COST Action MP0806 "Particles in turbulence". B.T. would like to acknowledge Université Libre de Bruxelles for being the hosting institution during the preparation of this work.

\section{References}

[1] R. J. McKay, K. G. McClements, A. Thyagaraja, and L. Fletcher, Plasma Phys. Control. Fusion 50, 065017 (2008).

[2] H. Abe, N. Sakairi, R. Itatani, and H. Okuda, JCP 63, 247 (1986).

[3] D. Tskhakaya, K. Matyash, R. Schneider, and F. Taccogna, Contributions to Plasma Physics 47, 563 (2007).

[4] P. Dmitruk, W. H. Matthaeus, N. Seenu, and M. R. Brown, Astrophys. J. Lett. 597, L81 (2003).

[5] P. Dmitruk, W. H. Matthaeus, and N. Seenu, Astrophys. J. 617, 667 (2004).

[6] R. I. McLachlan and G. R. W Quispel, J. Phys. A: Math. Gen. 39, 5251 (2006).

[7] H. Yoshida, Phys. Lett. A 150, 262 (1990).

[8] S. A. Chin, Phys. Rev E 77, 066401 (2008).

[9] E. Hairer, C. Lubich, and G. Wanner, Geometric Numerical Integration - Structure-Preserving Algorithms for Ordinary Differential Equations. Springer, 2nd edition, 2006.

[10] F. Mackay, R. Marchand, and K. Kabin, J. Geophys. Res. 111, 06208 (2006).

[11] F. Lekien and J. Marsden, Int. J. Numer. Meth. Engng, 63, 455 (2005).

[12] C.C. Lalescu, B. Teaca, and D. Carati, JCP 229, 5862 (2010).

[13] H. Homann, J. Dreher, and R. Grauer, Computer Physics Communications 177, 560 (2007).

[14] B. Teaca, M.K. Verma, B. Knaepen, and D. Carati, Phys. Rev. E 79, 46312 (2009).

[15] B. Teaca, C.C. Lalescu, B. Knaepen, and D. Carati, arXiv, physics.flu-dyn 1108.2640v1, (2011).

[16] S. Blanes and P.C. Moan, Journal of Computational and Applied Mathematics 142, 313 (2002). 